\title{
The Body Volume Index (BVI): Using 3D Scanners to Measure and Predict Obesity
}

\author{
Richard BARNES * \\ Select Research Ltd., Birmingham, UK
}

\section{Introduction}

Select Research is the leading company in the UK for the management of large-scale sizing surveys in the retail field, having recruited and scanned over 26,000 men, women and children for major UK retailers since 1997. This has included direct involvement in management of both 'national' sizing surveys in the UK for the retail industry.

Select were commissioned by UK retailers in 2001 to manage the recruitment and sampling for Size UK - the National Adult Survey as we had recruited and managed five sizing surveys for M\&S and Next before that. As a result of our experience, we were commissioned in 2008 by the retailers again to undertake the National Childrenswear Survey for them. This project is called Shape GB in recognition of the importance of body shape and to represent the geographical area of the representative sample.

However, in parallel to our extensive work in retail and in a totally different field, since the start of the millennium Select have been pioneering the use of 3D scanning technology in healthcare and specifically for the measurement of obesity with a new measurement; the Body Volume Index (BVI). The idea for BVI was originally conceived in January 2000, but development was delayed until we had completed our commitments to M\&S on their Bra Survey and the seventeen retailers on Size UK who commissioned us to help on that project in early 2001.

At that time, we recognised that the use of imaging such as MRI and DEXA have permeated healthcare and provide unparrelled data and insight into the condition of a patient, using leading edge technology to assist in the management of healthcare and patient care.

However, in contrast, risk assessment and measurement of obesity relied almost entirely on a manual measurement, the Body Mass Index (BMI), devised in 1830 as a broad based public health measurement tool. Measuring risk based only on height and weight, with no allowance for differences in gender, age or ethnicity, the use of manual techniques and the accuracy of BMI as a risk indicator has since been called into question $(1,2)$. Now, in the $21^{\text {st }}$ century, should we really still be so reliant on manual measurement for designation and evaluation of health risk?

Technology in healthcare is increasingly being used in measurement of an individual. However, in the initial stages of risk assessment for certain conditions such as obesity, healthcare professionals continue to remain reliant almost exclusively upon manual measurements. This is particularly true for obesity where manual measurements of waist circumference, waist to hip ratio and Body Mass Index (BMI) are currently used for assessment and diagnosis. However, recent published data (3, 4) have instigated a steady sea of change of opinion in the medical profession on the usefulness of $\mathrm{BMI}$ and prompted debate that a long-term replacement of BMI for the diagnosis of and health risk associated with obesity is necessary.

\section{History and Development}

\subsection{The origins of BVI}

In 1997 we were commissioned by Next to undertake a Womenswear Sizing Survey using 2D scanners and this led to 1999 to commissioning of four separate sizing surveys for Marks \& Spencer plc in conjunction with a company called CCR. Select successfully completed two previous sizing surveys with CCR for M\&S; Womenswear and Menswear before we were commissioned by Marks \& Spencer plc to undertake the Childrenswear Sizing Survey. The Childrenswear Survey consisted of the recruitment and measurement of 3,500 children aged 0-16 in the UK and we managed this project in conjunction with CCR.

\footnotetext{
* Select Research Ltd., 42 Calthorpe Road, Edgbaston, Birmingham, B15 1TS Tel. 00441886884009 richard@select-research.com
} 
The M\&S Childrenswear Sizing Survey was set up within eight weeks and involved measurement of children primarily in the school environment with some measurement of the 0-4 year olds undertaken using the interviewers employed by Select. The two previous adult surveys for M\&S had used 2D and 3D scanners supplied by Telmat and Wicks \& Wilson, but after much deliberation, it was decided that given the sensitivity of using scanners whereby children would have to undress, manual measurement using trained Nurses would be a more pragmatic and sensible approach for this survey.

Data collection for the survey began in late 1999 and during the initial phases of the project, it became apparent from media coverage of the sizing surveys that we had undertaken for M\&S that 'obesity' and 'childhood obesity' were public health issues, albeit not as prevalent and high profile as they are now. As a specialist in this field, I started to contemplate the options of using 3D scanners to measure obesity.

The non-use of 3D scanners on the children's survey and the importance and direct use of them on the previous three adult surveys highlighted the potential to me of using $3 \mathrm{D}$ scanners for measurement of obesity and health risk in both children and adults.

As a result of this, I authorised an internal research project after data collection had finished on the Childrenswear survey to investigate the potential use of 3D scanning technology for healthcare. We decided to research the concept with patients, General Practitioners and Hospital Doctors at the beginning of the millennium.

\subsection{Research and Development of the BVI concept}

The aforementioned research project commenced in mid January 2000 and involved a series of telephone depth interviews with patients and General Practitioners. The interviews were set up by our research team and conducted by me across the country to ascertain:-

- the awareness of 3D scanners given the high profile of the M\&S sizing surveys

- the then use of measurement within healthcare settings and for what purposes

- the potential uses of 3D scanning technology within healthcare settings

- the barriers and restrictions to usage given the prominence of the National Health Service (NHS) in the UK

- the existing use of the Body Mass Index (BMI) as a risk indicator in healthcare

Following these initial interviews, focus groups were set up in late January 2000 in Birmingham, UK amongst Nurses, General Practitioners (GP's) and Orthopaedic Consultants from a selection of local Hospitals. The focus groups lasted 1.5 hours and were open ended in terms of content to evaluate the potential uses and impact of using 3D scanners in healthcare. I moderated the focus groups at a viewing facility to allow for both filming and audio recording of the research.

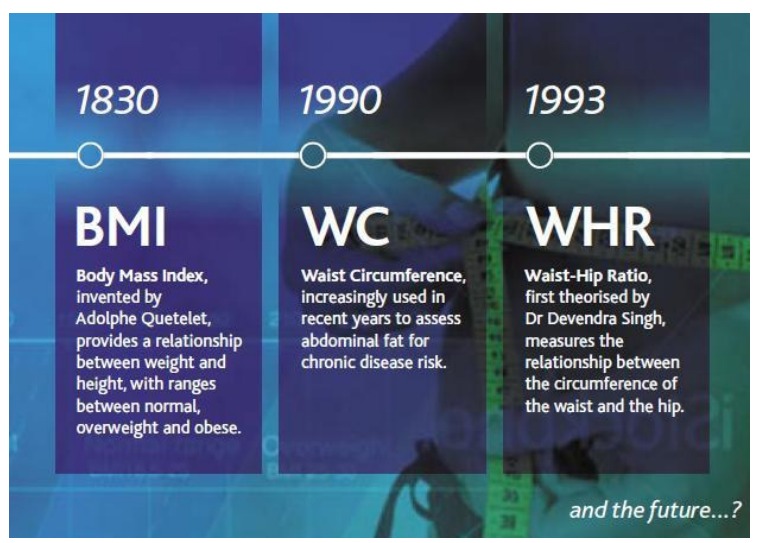

Fig. 1. The leaflet used to launch The Body Benchmark Study highlighting the current manual obesity measures. The public launch date for BVI was on the reverse 
From the research, it became apparent that there was a scope for 3D scanning technology in healthcare for specific applications such as prosthetics, osteoporosis, and physiotherapy. Moreover, it emerged that the respondents on the whole acknowledged that there was no cohesive and identifiable measurement process used within healthcare focussed on the individual health risks of a patient. The management and use of manual techniques such as the Body Mass Index (BMI), Waist Circumference and the recently publicised Waist to Hip Ratio were undertaken out of an obligation to national and international guidelines rather than as a conscious or rational choice.

In the following weeks, I listened again to recordings of the groups and it became immediately evident to me that there was a recognisable opportunity for a general improvement of the collection and delivery of measurement data within the NHS. Reviewing the options of using 3D scanners to assist and alleviate that need, I explored the options for using scanners to address what I believed was the key issue itself; namely the measurement processes and applications themselves. In short, I wondered if it would be possible to measure automatically as opposed to manually and if so, what would be the best and most simple means of measuring the human body in healthcare?

Using my fifteen years experience of applying research solutions to solve difficult marketing and product issues for other clients, I ascertained that a simple and easily understood measurement, like BMI was a pre-requisite. Although there was an undoubted acceptance and recognition from the respondents interviewed that 3D scanners could offer a new dimension to existing measurement, the realisation was that an 'add on' to an existing measurement was in itself a barrier to adoption. Manual measurement, being cost-free, quick and simple to use effectively meant that usage would in practice not change in the patient care pathway. 3D scanning if adopted as an 'extra' element would have the potential of increasing waiting times and costs to the NHS, both immediate barriers to adoption on a large-scale.

I therefore explored the possibility of creating a completely 'new' measurement; effectively to replace rather than augment the existing manual measurements and considered what might be the best option. During this evaluation I explored all options for measurement of the human body using a 3D scan and as a result theorised that measurement of volume, as opposed to linear measurement for apparel manufacture and design was probably the best option for creation of a new measurement. Effectively I decided that to measure the body in the reverse to retail (where external measurements are paramount) would be the best option for healthcare. In particular, I calculated that if 3D scanners could be adapted to measure part volumes of the body and in particular the abdominal volume of a patient's body that would give weight distribution; a key element to establishing genuine health risk.

I realised that measuring internal volume by body part could measure partial weight distribution and therefore for the first time, take the individual body shape of a patient into account when assessing their health risk. The idea for BVI was born and I immediately realised the potential impact of this were it to eventually be developed as a new measurement of obesity to enhance and possibly eventually replace manual methods of data collection. In order to simplify the understanding of what it was, I decided it had to be assigned a designation to emphasise both the link and the difference between the old and the new; therefore I decided on the term 'Body Volume Index' to define and describe the potential new measurement. The abbreviation (BVI) was a simple and effective comparator to the abbreviation for the Body Mass Index - BMI.

I continued to work on calculations and software options for BVI to ascertain how best the internal 'space' of the 3D scan could be filled in to ensure that Body Composition was both accurate and appropriate to healthcare. Development continued throughout 2000 and early 2001 but was then delayed due to the commissioning of the M\&S Bra Survey in 2000 and the Size UK project in 2001.

However, I continued to ponder how best to turn my idea into a reality and what would be the best means to achieve this. 


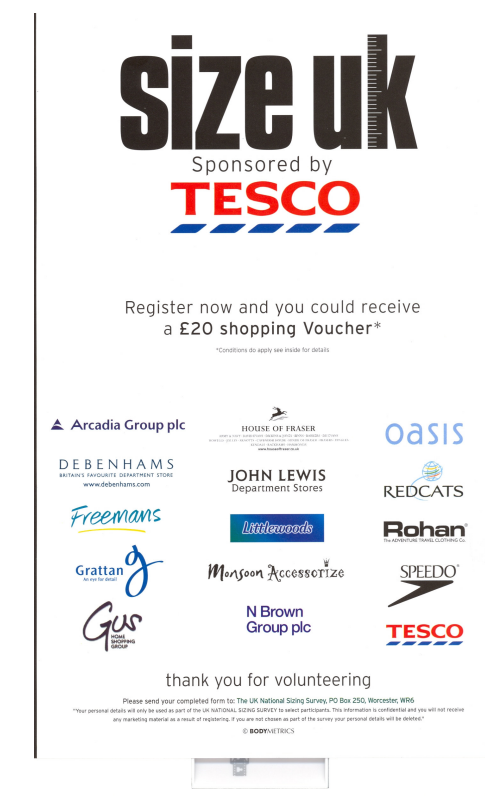

Fig. 2. The leaflet used by us to recruit 11,000 participants for the Size UK project

However, when our commitment on Size UK was complete in April 2004, the Chair of the Association for the Study of Obesity sent 350 letters to their members across the world inviting potential collaborators to join us in research and development of BVI. Thus commenced the preliminary research programme for BVI culminating in a public launch of The Body Benchmark Study on March $27^{\text {th }} 2007$ to formally announce BVI as a potential new standard for measurement of obesity and health risk with a team of internationally recognised healthcare experts.

\subsection{Additional research and validation}

We undertook qualitative research with healthcare practitioners in many disciplines which indicated that a 3D scan of the same patient taken for their BVI could potentially be used for review of anaesthetic dosage pre-operation, posture and osteoporosis issues, artificial limb construction and physiotherapy in addition to general health risks focussed on central (abdominal) obesity.

Further qualitative research undertaken by us in 2006 also highlighted that waiting times for operations could be reduced and more effective scheduling achieved by using BVI scans at preoperative level. BMI is the diagnostic reference point for the scheduling of patients pre-op on a daily basis, but using BVI offers the potential for patient scans to be assessed by an Anaesthetist in advance of theatre. Weight distribution (measured by BVI) is a key factor in deciding the length of time a patient might require in theatre and therefore which patients should be operated on first.

The 3D scanner operating system has been subjected to external analysis for epilepsy risk (5) given the use of white light, as this has been identified as the only possible risk to patients. Select have scanned over 28,000 men, women and children with no adverse side effects and the report concluded that 'in a normal adult population (over 18), the risk of photosensitivity is at most 1 in 20,000 .

The safety of the scanner and the scanning process are equally relevant with children as with adults. Between March 2009 and August 2010, Select Research scanned over 2,500 boys and girls aged 4-17 on Shape GB (6) with no adverse complaints from children or their parents. 


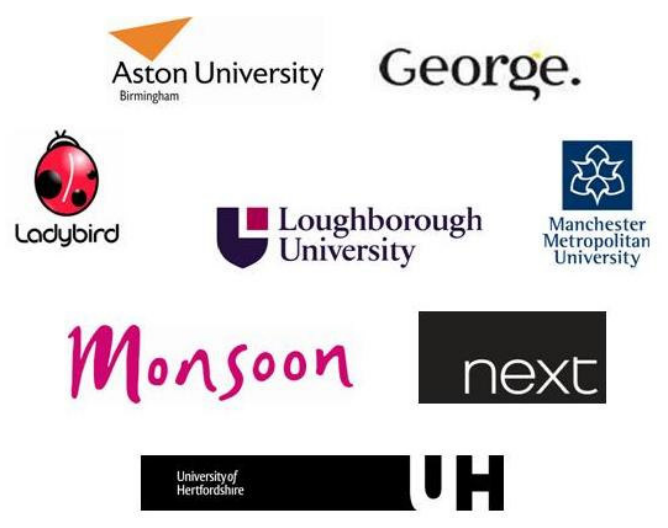

Fig. 3. The collaborators on Shape GB - The National Childrenswear Survey

This might be significant for a longer term review of the current National Child Measurement Programme (NCMP). The use of BVI may help alleviate some of the well publicised issues in the UK media regarding letters sent to parents about their supposedly obese children, where BMI has been incorrectly used as the reference point to determine whether a child is overweight or not.

Additional research (as yet not in the public domain) compares BVI with current techniques for body composition analysis, such as MRI, DEXA, and the correlation between body volume and cardiovascular health risk. The most comprehensive data of real body composition statistics in the world will be used exclusively as a baseline tool within BVI to ensure that empirical data is used as the cornerstone of the body composition element of BVI. Further validation, using CT, MRI, DEXA and Bio-Impedance, is currently being undertaken by the five healthcare collaborators in the Study in various independent and collaborative trials using the five 3D scanners set up for The Body Benchmark Study.

\section{$2.43 \mathrm{D}$ scanners and $\mathrm{BVI}$}

The Tc2 NX16 scanner was used as a baseline data collection tool using its combination of 32 cameras and 16 sensors to measure distance, using the combination of aperture, sensor, and reflective light, to extract the raw data. We were familiar with the Tc2 scanner from our work on Size UK but required sophisticated software to analyse part volume using the Body Composition data available to us, so new BVI software was developed to allow for segmental analysis of the human body and calculate body composition by body part to create a Health Risk Indicator. Health data including age, gender and ethnic origin and surrogate markers for cardiovascular disease such as lipid profile, blood pressure and heart rate were also included.

Using standard white-light data capture, Body Volume Imaging is non-invasive and uses white light reflected on the body to create an exact 'virtual' image of a person's shape. Up to 200 separate linear data measurements can also be extracted. The BVI Scanner is described as such as it measures the 'inside' or the 'volume' of the body as opposed to the scanners historically used in the retail field, which measure the outside for clothing.

The scanner can accurately measure people up to $205 \mathrm{~kg}$ in weight or up to a $150 \mathrm{cms}$ ( 60 -inch) waist circumference, so more than appropriate for all those except the most obese of patients. The BVI scanner, by its nature, cannot reduce energy output as BMI is a manual data collection method, but the scanner only requires a normal 13 amp socket to be operated so can be incorporated into a Health Centre with little disruption or the need to alter premises.

The process is completely harmless and the quality of data far supersedes the use of manual techniques where operator error and inconsistency produce greater variation of data. Validation by Mayo Clinic on the data output of the scanner (7) has indicated that in a direct comparison between manual and automatic measurement, there is less deviation from scanner measurements than from manual measurements of the same. Being fully automated for multiple measurements, BVI will help to reduce the printing and production of measurement data, which is relevant to the sustainability agenda in the National Health Service (NHS). 


\subsection{The significance of 'national' and 'benchmark' as reference criteria in sizing research}

The use of the words 'national' and 'benchmark' in the seven large sizing surveys are synonymous with the intention and the data sampling needs of the clients we are commissioned by.

Size UK in 2001 and Shape GB in 2009 were both sampled by us to ensure they were broadly representative of the national population and that experience gleaned from this work and my 25 years experience in market research enabled us to establish the most suitable reference for BVI.

Essentially BVI as an application has two reference points:-

The Patient - BVI is referenced to the average part volume distribution of their own peer group based on age, ethnicity and gender. They are compared to their own.

The Population - BVI records are effectively stored anonymously for public health purposes with a reference number that can identify the individual statistics for BMI, Waist Circumference, Waist to Hip Ratio, BVI, Ethnicity, Gender and Age. This allows for multi-faceted data analysis from a single data collection point and a single data source.

The above criteria ensures that BVI can effectively serve both as a one-stop reference point for anthropometric data in healthcare and as an individual patient record.

\section{Research and Validation}

\subsection{Research on BVI prior to the Body Benchmark Study}

A major new study, The Body Benchmark Study was launched in March 2007 to create the Body Volume Index (BVI); the world's first computer based anthropometric measurement for healthcare using 3D scanners that allows assessment of obesity based on body volume, body composition and body shape. Prior to that, over four years of preliminary work had been undertaken following the letter by ASO in September 2004.

Patients were consulted during extensive Qualitative Research undertaken by Select Research in 2006 and their insights used to formulate and create the delivery structure of the BVI application. Research was conducted amongst 85 patients using a series of focus groups and depth interviews in a viewing facility and the sample profile covered all ages, both genders and different socioeconomic groupings.

This research highlighted that patients acknowledged and understood the use of BMI as a measure of obesity, but were unaware of its definition and therefore unappreciative of it's limitations in measuring obesity. However, when presented with a scenario whereby BMI could be replaced by an alternative, the desire from respondents was for a simple and understandable measure, hence the emphasis on abdominal volume as a percentage of overall volume within BVI. All respondents understood the simple notion of 'the more weight around your stomach, the greater the health risk'.

Further quantitative research was undertaken over 6 months by researchers from the University of Warwick on the trial in 2006 (8) focussing on whether the 3D images had assisted individual patients in addressing their own weight and dietary issues. This was augmented by individual consultancy sessions with qualified Dieticians with each patient at 0,3 \& 6 month intervals. The results overall were inconclusive given the small sample size, but it was evident that some patients were motivated to lose more weight by managing their diet more effectively and that the key motivating factor for them had been the use of their previous 3D scans for comparative purposes. 


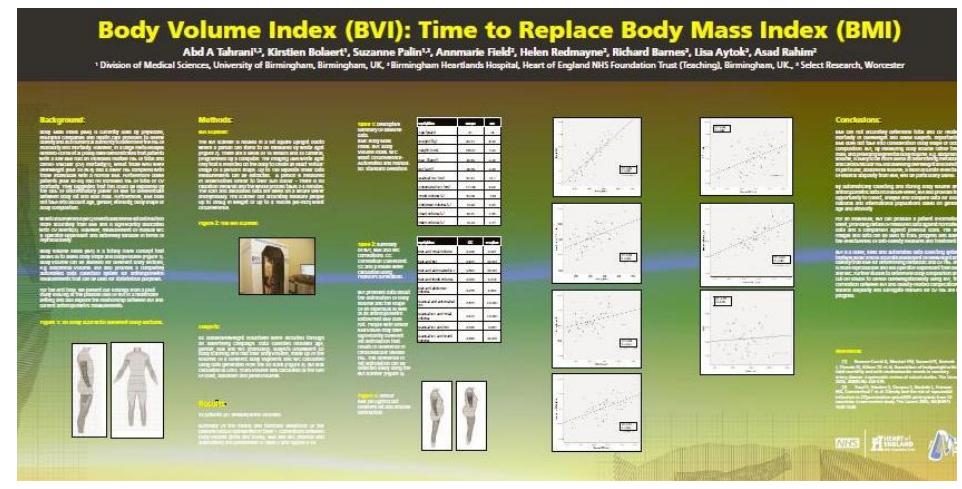

Fig. 4. The published abstract for the 2008 Weight Management trial by Heartlands

A prototype version of BVI was developed using the initial calculations from 2000 and 2001 and on the principle that abdominal volume, the section of the body identified as being crucial for diagnosis of central obesity, was attributed with an importance factor. The BVI calculus, algorithms and ratios were planned to interact to provide a predictive risk factor based on the level of abdominal volume (as a percentage of the total body) taking at least age, ethnic origin and gender into account. BMI does not accommodate this level of differentiation. Chest volume was also analysed to ensure that people of athletic build (but with a high BMI) are designated with an appropriate BVI figure and thigh and leg volume were also contributing factors in any BVI value.

\subsection{The Body Benchmark Study}

Despite this extensive work on BVI, we realised that to ensure widespread adoption and acceptance of BVI, healthcare professionals across the world in appropriate disciplines would be required to validate BVI long-term. Therefore, The Body Benchmark Study was set up as a collaboration between world-renowned healthcare research facilities and hospitals in the US, the United Kingdom and mainland Europe. Some of these such as Heartlands Hospital in the UK and Mayo Clinic in Rochester, Minnesota, USA have published data and other healthcare specialists in child obesity, diabetes, body composition and risk assessment throughout the world will be doing so at other periods during the next two years.

The main objectives of the Body Benchmark Study were:-

1. To validate BVI against established manual measurement alternatives such as BMI, Waist/Hip ratio and Waist Circumference and volume measurement devices such as The Bodpod.

2. To automate data collection of body measurements and to allow for national and international statistical analysis of BVI by age, gender, ethnicity and body shape.

3. To establish a series of ratios and relationships between part volumes created by a BVI scan, e.g. abdominal volume, and correlate these with surrogate markers for cardiovascular health risk.

4. To validate health risk for an individual by taking into account body shape, distribution of body volume and fat, and age, gender and ethnicity.

5. To integrate body composition and body scan data by direct means from cadaver analysis for total and body part volumes.

The published results of the Body Benchmark Study were presented at a major media launch set up on October $12^{\text {th }}$ 2010. Details of the launch and the published results can be found on www.bodyvolume.com . 


\subsection{Government evaluation of obesity and the costs to the NHS}

The costs of obesity and associated health risks to the NHS are expected to rise to £3.6billion a year by 2010 and HM Government's Foresight report (9) suggests that if current trends continue, almost 9 in 10 adults and two-thirds of children will be obese by 2050, resulting in an overall cost to society of $£ 50 \mathrm{bn} /$ annum (10). It is anticipated that BVI will assist in reducing some of these costs.

\section{Results}

\subsection{Initial results from the Body Benchmark Study}

Four trials have been published by Heartlands Hospital (UK) and Mayo Clinic (USA) and further research in four trials has been completed but is not yet in the public domain. Initial results indicate that with BVI, the long-term monitoring of changes in an individual's body shape may encourage individual weight loss and help with the review of national strategies for obesity management (11).

Individually tailored preventative and diagnostic treatment appropriate to a patient's health needs is possible as BVI will offer more accurate information about their history of obesity using 3D data and images. Now, research undertaken during the Heartlands Hospital trial on weight motivation ( 8, 12 ) has established that some individuals were motivated by reference to their own previous scans and that their own age, gender and ethnicity were key factors in establishing their own perception of weight and the need to address that through nutritional and diet changes.

BVI provides data about distribution of body volume, body fat and the shape of an individual, something BMI can never do (Figure 5). BVI is more accurate in 'contour' measurement of an individual than BMI or a tape measure can be as it measures the actual skin surface of the waist area, which includes the indent at the small of a person's back where the spine is.

Recent validation by Heartlands NHS Hospital in the UK $(8,12)$ and Mayo Clinic in the US $(7,13)$ have highlighted the benefits of using the BVI Scanner and the better accuracy of measurement generated when compared to manual measurements of waist and hip (7). These studies illustrate the benefits of using a scanner for repeatability of measurements in comparison to manual measurement and in particular where part volumes can highlight where volume is and therefore where weight is located. Volume is defined as the space that an object occupies and the interior of the human body has components in each body section which are unique in content, but more importantly in volume and weight. which BVI can calculate.

The research has shown that BVI offers many advantages over the more traditional BMI, and that BVI can be used to assess body shape, provide automated anthropometric measurements and track changes in body shape over time. This is something hitherto not possible - using BVI to effectively predict and estimate obesity levels of an individual patient based on their variations in body shape over time.
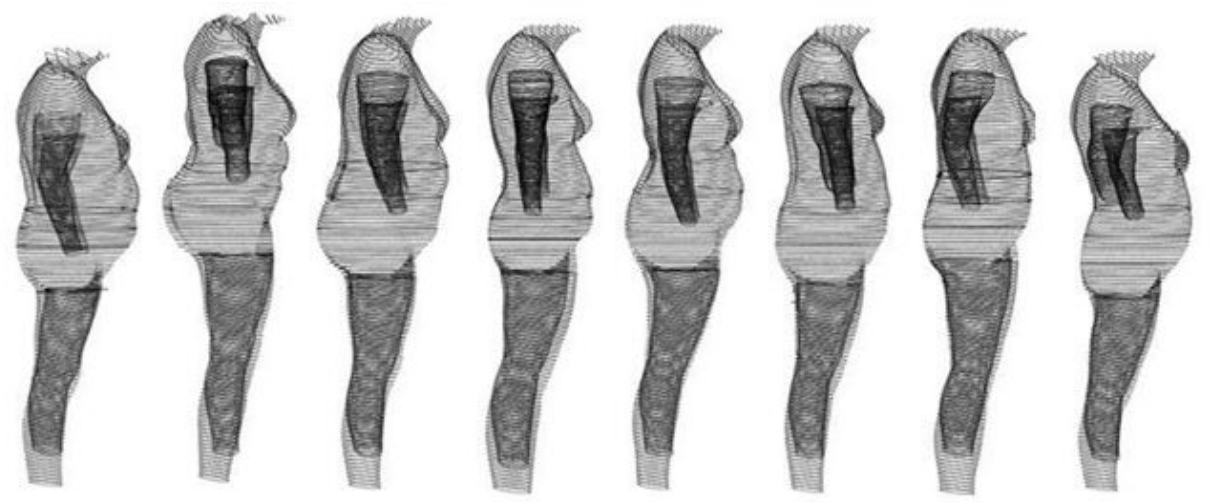

Fig. 5. 8 women with a BMI of 30 the threshold for obesity highlighting different body shape 
The preliminary data from this trial has shown that part body volumes can be tracked in a patient and that changes, in loss or gain, can be automatically recorded and analysed by the scanner. By using techniques like BVI we can spot obesity problems early and manage them earlier.

\subsection{How does BVI work?}

BVI by taking and comparing the part-volumes of a 3D scan can:

- Automatically measure the changes in a person's body volume and part volume

- Highlight the risks associated with greater or less volume in a given region, for example the abdominal area

- Relate the health risks of a person to their body shape, taking medical history, surrogate markers for cardiovascular disease, e.g. lipid profile, C-reactive protein, age, gender and ethnicity into account

- Compare a patient's BVI against normative data of shape for their age, gender and ethnic grouping.

Data from scans can be compared with normative data or merged to create an 'average' shape of a particular age, gender, ethnic group or even whole population. This has tremendous implications when measuring national and international obesity levels over time.

There are other possible applications for BVI within healthcare such as physiotherapy, reconstructive surgery, eating disorders and prosthetics. The linear measurements can also be programmed to assist in the development of orthopaedic supports based on individual shapes of patients and diagnosis of body shape change over time.

\subsection{What are the benefits of BVI?}

BVI is accurate, reproducible and not operator dependant. Despite World Health Organisation (WHO) guidelines on performing waist measurements, the reality of the manner in which people are measured manually is highly variable and a real issue in practice.

Non-medical administrative staff can operate BVI scanners and as a first stage assessment tool, the benefits are enormous. MRI scanners are infinitely more sophisticated in terms of individual assessment and diagnosis of internal organs and possible health factors that would affect a patient. However, MRI as a population based screening tool is counter productive, both cost wise and in terms of speed and convenience for a patient. The BVI Scanner is meant to provide an alternative to $\mathrm{BMI}$ as a risk assessment mechanism for an individual patient and be a cost-effective tool for public health purposes.

The BVI system is an alternative to BMI and will provide benefits to healthcare in the following areas:-

1. As an obesity assessment tool, BVI will allow for quick, safe and accurate assessment of body weight, body composition, fat distribution and anthropometric measurement of an individual. This data can then be used for the health risk assessment of individuals and offer clinicians a useful assessment tool to discuss sensitive body shape issues with a patient and to use the data as a motivational tool for obesity and other health issues (Figure 6). 

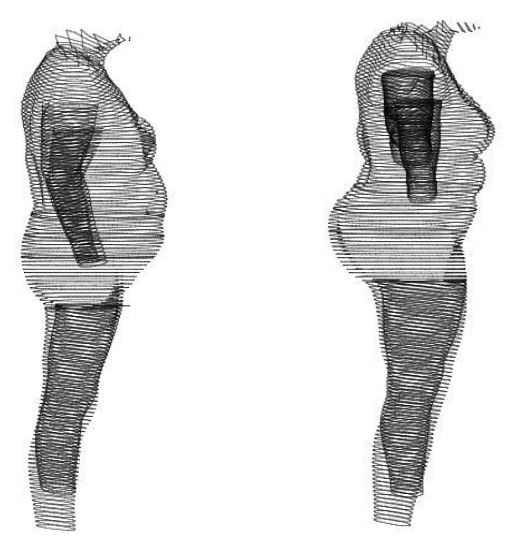

Fig 6. BMI does not allow differentiation between two individuals with exactly the same BMI (>30) but different weight distribution.

2. BVI will produce a patient information sheet, providing details of measurement data against normative data and comparison against previous scans for the gender, age and ethnicity of that patient.

3. BVI will provide an automated data collection system for individuals, local, national and international population statistics.

4. BVI will assist in the administration of certain drugs, e.g. anaesthetics; can be based on body volume and surface area rather than whole body weight.

\section{Conclusions}

BVI is the world's first dedicated measurement standard for obesity and body shape analysis using computers, capable of assessing populations as well as individuals. Rather than one standard measurement technique for all people, BVI is based on individual body shape and able to divide the 3D body image of an individual into multiple body volumes, so part body composition can be analysed, allowing differentiation between people with the same BMI.

The deliverable is the first computerised anthropometric measuring system for obesity and initial health risk. Applications will include individual assessment of obesity and related health risks and a fully computerised system for the collection and tracking of individual, local, national and international obesity statistics.

BMI is nearly 200 years old will still have a place and use in healthcare. However, in the computer age, for individual obesity, a manual 'good guess' is simply not good enough and reliance upon $\mathrm{BMI}$ is not conducive to the good of the patient and healthcare management in the long-term.

The Body Benchmark Study has hopefully helped to provide the first step in creating a new measurement more appropriate and meaningful for the modern world - where tracking and recording of an individual's body shape and weight distribution will ultimately allow for predictive analysis of that person's potential obesity and diagnostic needs in the future.

In early 2011, Mayo Clinic will present their findings on twelve part volume indices for BVI. Following three years of research, they have drawn conclusions on the most appropriate 'index' for BVI which best indicates the cardiometabolic risk of an individual patient and the added value that BVI offers over current manual methods of measurement in healthcare. This will provide the next development stage in BVI as a long-term measure of obesity and help BVI to become a significant new point of reference for healthcare. 


\section{References}

1. Sebo P, beer-Borst S, Haller DM et al. Reliability of doctors' anthropometric measurements to detect obesity. Preventive Medicine 2008.

2. Nadas J, Putz Z, Kolev G et al. Intraobserver and interobserver variability of measuring waist circumference. Medical Science Monitor 2008; 14:CR15-18.

3. A. Romero-Corral, V. K. Somers, J. Sierra-Johnson, R. J. Thomas, M. L. Collazo-Clavell, J. Korinek, T. G. Allison, J. A. Batsis, F. H. Sert-Kuniyoshi \& F. Lopez-Jimenez (June 2008).

"Accuracy of body mass index in diagnosing obesity in the adult general population". International Journal of Obesity 32 (6): 959-956. doi:10.1038/ijo.2008.11. PMID 18283284.

4. A. Romero-Corral et al; Association of bodyweight with total mortality and with cardiovascular events in coronary artery disease: a systematic review of cohort studies." Lancet (2006-0819;368(9536):666-78). Retrieved on 2008-09-08

5. Radtke R.A., NX12 Body Measurement System; Assessment of Risk for Photosensitive Seizures, Duke University Medical Center, Durham North Carolina, USA, February 2006

6. Shape GB - The National Childrenswear Survey; www.shapegb.org

7. Korenfeld $Y$, Ngwa T, Friedman $L$ et al. Validation of a Novel 3D Body Scanner for Obesity Anthropometric Measurements AHA $26^{\text {th }}$ Princeton Conference on Cerebrovascular Disease, Houston, Texas, USA March 2009.

8. Boelaert $\mathrm{K}$, Palin S, Field $A$ et al. The impact of $3 \mathrm{D}$ body images on motivating weight loss in overweight individuals. Endocrine Abstracts 2008; 15:125.

9. Foresight Tackling Obesities: Future Choices - Project Report. Government Office for Science 2007.

10. Hospital Episode Statistics Online 2005-2006.

http://www.hesonline.nhs.uk/Ease/servlet/ContentServer?sitelD=1937\&categorylD=245

11. Healthy Weight, Healthy Lives: a cross-government strategy for England. Cross-Government Obesity Unit, Department of Health and Department of Children, Schools and Families. London, January 2008.

12. Tahrani $A$, Boelaert $K$, Barnes $R$ et al. Body volume index: time to replace body mass index? Endocrine Abstracts 2008; 15:104.

13. Romero-Corral A, Somers VK, Lopez-Jimenez F et al. 3-D body scanners - body volume index: a novel, reproducible and automated anthropometric tool associated with cardiometabolic biomarkers. Conference Abstract. The Obesity Journal 2008; 16:S124.

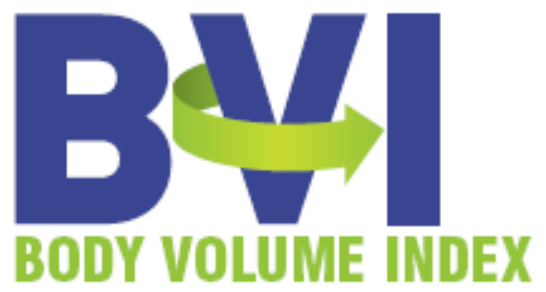

